\title{
Orbital infantile hemangioma and rhabdomyosarcoma in children: differentiation using diffusion-weighted magnetic resonance imaging
}

Stephen F. Kralik, MD, ${ }^{a}$ Kathryn M. Haider, MD, ${ }^{b}$ Remy R. Lobo, MD,${ }^{c}$ Nucharin Supakul, MD, ${ }^{\text {a }}$ Sonia F. Calloni, MD, ${ }^{\mathrm{d}}$ Bruno P. Soares, $\mathrm{MD}^{\mathrm{e}}$

Author affiliations: ${ }^{a}$ Department of Radiology and Imaging Sciences, Indiana University School of Medicine, Indianapolis; ${ }^{b}$ Department of Pediatric Ophthalmology, Riley Hospital for Children, Indianapolis, Indiana; ${ }^{c}$ Neuroradiology Division. Department of Radiology, University of Michigan Health System. Ann Arbor; ${ }^{d}$ Universita degli Studi di Milano, Postgraduation School in Radiodiagnostics, Milan, Italy; ${ }^{e}$ Section of Pediatric Neuroradiology, Division of Pediatric Radiology, Russel H. Morgan Department of Radiology and Radiological Science, Johns Hopkins University School of Medicine, Baltimore, Maryland

Submitted April 24, 2017.

Revision accepted September 4, 2017.

Correspondence: Stephen F. Kralik, MD, 714 N. Senate Ave Suite 100, Indianapolis, IN, 46202 (email: steve.kralik@gmail.com).

Word count: 2,169

Abstract only: 262

This is the author's manuscript of the article published in final edited form as:

Kralik, S. F., Haider, K. M., Lobo, R. R., Supakul, N., Calloni, S. F., \& Soares, B. P. (2017). Orbital infantile hemangioma and rhabdomyosarcoma in children: differentiation using diffusion-weighted magnetic resonance imaging. Journal of American Association for Pediatric Ophthalmology and Strabismus. 


\section{Abstract \\ Purpose}

To evaluate differences in magnetic resonance imaging (MRI) appearance between infantile hemangiomas and rhabdomyosarcomas of the orbit in pediatric patients using diffusion-weighted imaging.

\section{Methods}

A multicenter retrospective review of MRIs of pediatric patients with infantile hemangiomas and rhabdomyosarcomas of the orbit was performed. MRI examinations from a total of 21 patients with infantile hemangiomas and 12 patients with rhabdomyosarcomas of the orbit were independently reviewed by two subspecialty board-certified neuroradiologists masked to the diagnosis. A freehand region of interest was placed in the mass to obtain the mean apparent diffusion coefficient (ADC) value of the mass as well as within the medulla to obtain a ratio of the ADC mass to the medulla. A $t$ test was used to compare mean ADC and ADC ratios between the two groups. Receiver operating characteristic analysis was performed to determine ADC value and ADC ratio thresholds for differentiation of infantile hemangioma and rhabdomyosarcoma.

\section{Results}

There was a statistically significant difference in the mean ADC value of infantile hemangiomas compared to rhabdomyosarcomas $\left(1527 \times 10^{-6} \mathrm{~mm}^{2} / \mathrm{s}\right.$ vs $\left.782 \times 10^{-6} \mathrm{~mm}^{2} / \mathrm{s} ; P=0.0001\right)$ and the ADC ratio of the lesion to the medulla (1.77 vs 0.92; $P=0.0001)$. An ADC threshold of $<1159 \times$ $10^{-6} \mathrm{~mm}^{2} / \mathrm{sec}$ and an ADC ratio of $<1.38$ differentiated rhabdomyosarcoma from infantile hemangioma (sensitivity $100 \%$ and $100 \%$; specificity $100 \%$ and 100\%) with area under the curve of 1.0 and 1.0, respectively. 


\section{Conclusions}

In conjunction with conventional MRI sequences, ADC values obtained from diffusion-weighted MRI are useful to differentiate orbital infantile hemangiomas from rhabdomyosarcomas in pediatric patients. 
Magnetic resonance imaging (MRI) is the imaging modality of choice for pediatric patients presenting with an orbital mass. Pediatric orbital masses may involve a wide range of benign and malignant pathologic diagnoses, including infantile hemangioma, venous and lymphatic malformations, epidermoid cyst, Langerhans cell histiocytosis, metastatic disease, optic nerve glioma, and rhabdomyosarcoma. Conventional MRI, including T1-weighted (T1W), T2weighted $(\mathrm{T} 2 \mathrm{~W})$, and contrast-enhanced $\mathrm{T} 1$-weighted $(\mathrm{T} 1 \mathrm{~W}+\mathrm{C})$ sequences are able to narrow the differential diagnosis or indicate a specific diagnosis based on location and imaging appearance; however, some masses may have overlapping imaging features. Because malignant tumors frequently are associated with increased cellularity, reduced extracellular space, and larger nuclei, a corresponding dark signal intensity on apparent diffusion coefficient (ADC) mapping frequently correlates with high-grade or malignant pathology. ${ }^{1-4}$ Previous studies have indicated that lower ADC values can be useful for differentiating benign and malignant neck and orbital lesions. ${ }^{5-7}$

Rhabdomyosarcomas are the most common soft tissue sarcoma in children, with peak incidence occurring in patients 0-4 years of age and the majority of malignant head-and-neck tumors in the orbit. ${ }^{8}$ Conversely, infantile hemangiomas are the most common benign neoplasm in infancy, with the majority occurring in the first year of life. Infantile hemangioma and rhabdomyosarcoma may have similar appearance on the T2W and T1 W+C MRI sequences, and age at presentation may overlap, resulting in clinical and radiological diagnostic uncertainty. A previous study reported suggested that diffusion-weighted imaging (DWI) could differentiate infantile hemangioma from rhabdomyosarcoma in the orbit among children; however, only 8 total patients with infantile hemangioma and rhabdomyosarcomas were included, only 4 had ADC images, and no ADC measurements were reported. ${ }^{9}$ Because treatment is significantly 
different for infantile hemangiomas compared to rhabdomyosarcomas, it is necessary to confirm whether MRI can reliably differentiate these masses. The purpose of this research was to evaluate a larger group of pediatric patients with infantile hemangiomas and rhabdomyosarcomas of the orbit to determine whether ADC can reliably differentiate these lesions.

\section{Subjects and Methods}

With institutional board review approval, a multicenter retrospective study from December 2008 to January 2016 identified pediatric patients (defined as age $<18$ years) with infantile hemangiomas and rhabdomyosarcomas of the orbit. Rhabdomyosarcoma pathology was confirmed in all patients on biopsy by a board-certified pathologist. Infantile hemangiomas were diagnosed by biopsy or by clinical follow-up in conjunction with the MRI in all patients by a board-certified ophthalmologist $(\mathrm{KH})$. Clinical follow-up for patients with infantile hemangiomas varied based on age of the patient and presence of ocular complication. Patients $<3$ months of age were generally followed every 1-3 weeks, whereas older patients are followed at longer intervals, depending on the rate of growth or stability.

MRI was performed on $1.5 \mathrm{~T}$ or $3 \mathrm{~T}$ MR imaging units. MRI protocol included a minimum of axial and coronal $\mathrm{T} 2 \mathrm{~W}$ fat saturation, axial and coronal $\mathrm{T} 1 \mathrm{~W}$ precontrast, $\mathrm{T} 1 \mathrm{~W}+\mathrm{C}$ with fat saturation, and axial DWI sequence. DWI sequences were performed with single-shot spin-echo echo-planar imaging with 5-mm section thickness before administration of contrast material, with $\mathrm{b}$-values of 0 and $1000 \mathrm{~s} / \mathrm{mm}^{2}$ applied in the $\mathrm{x}, \mathrm{y}$, and $\mathrm{z}$ directions. Orbital location was defined as involvement of either the preseptal or postseptal orbit on MRI. Patients were excluded if no MRI was available, if any of the $\mathrm{T} 2 \mathrm{~W}, \mathrm{~T} 1 \mathrm{~W}+\mathrm{C}$, or DWI sequences were absent or distorted by artifact. Subspecialty board-certified neuroradiologists (SK, BS) with at least 4 years of 
clinical experience performed individual retrospective reviews of the MRIs on the PACS while masked to the diagnosis and recorded the qualitative $\mathrm{T} 2 \mathrm{~W}, \mathrm{~T} 1 \mathrm{~W}+\mathrm{C}$, and $\mathrm{ADC}$ appearance for all masses. T2W was used to describe the appearance of the mass as hypointense, isointense, or hyperintensity relative to normal brain parenchyma and to determine the presence or absence of flow voids, defined as linear hypointense signal in the mass. $\mathrm{T} 1 \mathrm{~W}+\mathrm{C}$ was used to determine either homogenoeous or heterogeneous enhancement depending on the uniformity of the enhancement. The ADC images were used to describe the appearance of the mass as hypointense, isointense, or hyperintensity relative to normal brain parenchyma. The mean ADC value of the orbital mass was obtained by placing a freehand region of interest (ROI) on the ADC image at the level of the largest tumor diameter with sparing of the edge of the mass to avoid inclusion of normal tissue. The mean ADC of the medulla was used as an internal reference to calculate a ratio of the ADC value of the mass to the ADC value of the medulla. The medulla was chosen as an internal reference because it is reproducibly identified on MRI, it provides a precise location, and because its ADC value would be less affected by changes in myelination in children. The mean ADC value of the medulla was similarly obtained by placing a freehand ROI in the medulla on the ADC image, with sparing the edges of the medulla.

A $t$ test was used to compare the two group mean ADC values, mean ADC ratios, maximum diameter of the lesions, and mean age of patients. A $P$ value of $\leq 0.05$ was considered statistically significant. A receiver operating characteristic (ROC) curve was used to analyze threshold calculations. The statistical analysis of data was done using GraphPad Prism version 7 for Mac (GraphPad Software, La Jolla, CA).

\section{Results}

A total of 21 children with orbital infantile hemagiomas (median age, 5 months; range, 1-34 
months; 63\% female) and 12 patients with orbital rhabdomyosarcomas (median age, 57 months; range 20-281 months; 50\% female) were included. Five patients were excluded either because the ADC images were distorted by artifact or DWI was not performed. There was a statistically significant difference between the mean age of patients with orbital infantile hemangiomas versus rhabdomyosarcomas $(7.3 \pm 7.3$ months vs $103 \pm 91$ months; $P=0.0001)$. There was a statistically significant difference between the mean diameter of orbital infantile hemangiomas versus rhabdomyosarcomas $(2.5 \pm 0.8 \mathrm{~cm}$ vs $3.6 \pm 1.3 \mathrm{~cm} ; P=0.005)$. Rhabdomyosarcoma pathology subtypes consisted of 7 alveolar types, 3 embryonal types, and 2 that were not specified.

MRI appearance on $\mathrm{T} 2 \mathrm{~W}, \mathrm{~T} 1 \mathrm{~W}+\mathrm{C}$, and $\mathrm{ADC}$ sequences of the orbital infantile hemangiomas and rhabdomyosarcomas is seen in Table 1. Representative examples are provided in Figures 1-4. Qualitatively, infantile hemangiomas demonstrated T2W hyperintensity, homogeneous enhancement, presence of flow-voids, and ADC hyperintensity in 100\%, 100\%, $100 \%$, and $100 \%$ compared to rhabdomyosarocomas, which were $58 \%, 58 \%, 25 \%$, and $0 \%$, respectively.

There was a statistically significant difference between the mean ADC of infantile hemangiomas versus the rhabdomyosarcomas $\left(1527 \pm 173 \times 10^{-6} \mathrm{~mm}^{2} / \mathrm{s}\right.$ vs $782 \pm 127 \times 10^{-6}$ $\left.\mathrm{mm}^{2} / \mathrm{s} ; P=0.0001\right)$. There was a statistically significant difference between the ADC ratio of infantile hemangiomas versus the rhabdomyosarcomas $(1.77 \pm 0.23$ vs $0.92 \pm 0.21 ; P=0.0001)$. The ROC demonstrated a threshold ADC value for differentiation between orbital infantile hemangioma and rhabdomyosarcoma was $1159 \times 10^{-6} \mathrm{~mm}^{2} / \mathrm{s}$ with sensitivity of $100 \%$ and specificity of $100 \%$. The ROC demonstrated a threshold ADC ratio for differentiation between orbital infantile hemangioma and rhabdomyosarcoma was 1.38 , with a sensitivity of $100 \%$ and 
specificity of $100 \%$. Area under the curve was 1.0 for ADC and 1.0 for ADC ratio.

\section{Discussion}

This multicenter study of a large group of pediatric patients demonstrates that ADC appearance and quantification can reliably differentiate orbital infantile hemangiomas and rhabdomyosarcomas. As did Lope and colleagues, ${ }^{9}$ we demonstrate that rhabdomyosarcomas and infantile hemangiomas of the orbit may have similar $\mathrm{T} 2 \mathrm{~W}$ and $\mathrm{T} 1 \mathrm{~W}+\mathrm{C}$ appearances. ADC values of orbital rhabdomyosarcomas were found to be significantly lower than infantile hemangiomas, which is similar to findings from previous reports of smaller numbers of patients with infantile hemangiomas and rhabdomyosarcomas. ${ }^{5,6,10}$ Rhabdomyosarcomas demonstrated a mean ADC value of $782 \times 10^{-6} \mathrm{~mm}^{2} / \mathrm{s}$, which is similar to $720 \times 10^{-6} \mathrm{~mm}^{2} / \mathrm{s}$ reported in a metaanalysis of 12 rhabdomyosarcomas of the orbit. ${ }^{10}$ In conjunction with the conventional MRI sequences, ADC images appear to be helpful in differentiation of infantile hemangiomas and rhabdomyosarcomas, which can guide which patient should undergo biopsy and prevent delays in diagnosis.

We chose to evaluate rhabdomyosarcomas of the orbit because this is the most common location for rhabdomyosarcoma in the head and neck and most likely to result in diagnostic confusion with an infantile hemangioma. We chose to evaluate pediatric orbital infantile hemangiomas and rhabdomyosarcomas rather than all pediatric orbital lesions because the conventional MRI appearance often overlaps. Furthermore, inclusion of other pediatric orbital lesions, such as retinoblastoma or Langerhans cell histiocytosis was not justified, because those do not pose a diagnostic dilemma. Previous studies have attempted to aggregate benign and malignant orbital lesions, but this ignores the valuable input that $\mathrm{T} 2 \mathrm{~W}$ and $\mathrm{T} 1 \mathrm{~W}+\mathrm{C}$ imaging and tumor location have on narrowing a differential diagnosis. ${ }^{5,6}$ Selective usage of ADC information 
in conjunction with the clinical information (including the age of the patient) and conventional MRI sequences reflects routine clinical care and reflects more optimal usage of the ADC information. For example, although an epidermoid cyst demonstrates ADC hypointensity, it is easily differentiated from rhabdomyosarcoma by its lack of enhancement. Neuroblastoma and Langerhans cell histiocytosis in the orbit typically arise from osseous structures allowing differentiation from infantile hemangiomas and rhabdomyosarcomas. Similarly, orbital lymphoma demonstrates ADC hypointensity with ADC values with similar range as rhabdomyosarcomas; however, it is a rare diagnosis in children. ${ }^{5,6,-11}$ Therefore, selective use of $\mathrm{ADC}$ as a problem-solving tool is a more effective attempting to differentiate all benign and malignant lesions of the orbit.

Patient age also demonstrated a statistically significant difference between the two groups, which can be helpful in differentiation. Incidence of rhabdomyosarcoma peaks between 5 and 10 years of age, unlike infantile hemangioma; however, rhabdomyosarcomas have been reported in patients younger than 1 year of age. This suggests that differentiation based solely on age may result in diagnostic errors.

This study has several strengths, including multicenter evaluation of infantile hemangiomas and rhabdomyosarcomas, multiple imaging reviewers, and larger number of patients than reported in previous series. The robust nature of the ADC measurement is indicated by the demonstration that $\mathrm{ADC}$ values and $\mathrm{ADC}$ ratios for these lesions are similar across different institutions and MRI scanners as well as reproducible with different radiologists performing measurements. ADC ratio proved to be reliable and offers an alternative means to evaluate the two entities. The medulla was chosen because it is a precise anatomic location allowing reproducible ROI placement which is similar to use of the spinal cord as a reference 
location. ${ }^{12}$

We acknowledge potential limitations to this study. Infantile hemangiomas were verified by clinical follow-up in conjunction with imaging appearance rather than by pathologic diagnosis. However, requirement of a biopsy to diagnose an infantile hemangioma is not the standard of clinical care and is reserved for a select number of patients. A selection bias may lead to some infantile hemangiomas undergoing MRI; however, in many children infantile hemangioma cannot be diagnosed by clinical assessment alone. Another limitation is that some patients with infantile hemangioma were excluded because of DWI artifact. Artifact from magnetic susceptibility effects related to the bone is a known limitation of DWI. Advancements in DWI technology through readout-segmented echo-planar imaging have been described resulting in less anatomic distortion, and future studies using these newer techniques may be valuable. ${ }^{13}$ No patients with rhabdomyosarcomas were excluded because of DWI artifact, suggesting that most rhabdomyosarcomas are large enough at presentation to be adequately imaged with standard DWI technique. Lastly, due to the relatively small number of patients with rhabdomyosarcomas and 2 patients without pathologically specified categorization of subtype, subgroup analysis of pathology subtypes of rhabdomyosarcomas could not be performed. 


\section{References}

1. Humphries P, Sebire N, Siegal M, Olsen ØE. Tumors in pediatric patients at diffusionweighted MR imaging: apparent diffusion coefficient and tumor cellularity. Radiology 2007;245:848-54.

2. Guo AC, Cummings TJ, Dash RC, Provenzale JM. Lymphomas and high-grade astrocytomas: comparison of water diffusibility and histologic characteristics. Radiology 2002;224:177-83.

3. Kono K, Inoue Y, Nakayam K, et al. The role of diffusion-weighted imaging in patients with brain tumors. Am J Neuroradiol 2001;22:1081-8.

4. Sugahara T, Korogi Y, Kochi M, et al. Usefulness of diffusion-weighted MRI with echoplanar technique in the evaluation of cellularity of gliomas. J Magn Reson Imaging 1999;9:53-60

5. Sepahdari AR, Aakalu VK, Setabutr P, Shiehmorteza M, Naheedy JH, Mafee MF. Indeterminate orbital masses: restricted diffusion at MR imaging with echo-planar diffusion-weighted imaging predicts malignancy. Radiology 2010;256:554-64.

6. Abdel Razek AA, Elkhamary S, Mousa A. Differentiation between benign and malignant orbital tumors at 3-T diffusion MR-imaging. Neuroradiology 2011;53:517-22.

7. Abdel Razek AA, Gaballa G, Elhawarey G, Megahed AS, Hafez M, Nada N. Characterization of pediatric head and neck masses with diffusion-weighted MR imaging. Eur Radiol 2009;19:201-8.

8. Ognjanovic S, Linabery AM, Charbonneau B, Ross JA. Trends in childhood rhabdomyosarcoma incidence and survival in the United States, 1975-2005. Cancer 2009;115:4218-26. 
9. Lope LA, Hutcheson KA, Khademian ZP. Magnetic resonance imaging in the analysis of pediatric orbital tumors: utility of diffusion-weighted imaging. J AAPOS 2010;14:25762.

10. Sepahdari AR, Politi LS, Aakalu VK, Kim HJ, Razek AA. Diffusion-weighted imaging of orbital masses: multi-institutional data support a 2-ADC threshold model to categorize lesions as benign, malignant, or indeterminate. AJNR Am J Neuroradiol 2014;35:170-75.

11. Politi LS, Forghani R, Godi C, et al. Ocular adnexal lymphoma: diffusion-weighted MR imaging for differential diagnosis and therapeutic monitoring. Radiology 2010;256:56574.

12. Kolff-Gart AS, Pouwels PJ, Noij DP, et al. Diffusion-weighted imaging of the head and neck in healthy subjects: reproducibility of ADC values in different MRI systems and repeat sessions. AJNR 2015; 36:384-90.

13. Yeom KW, Holdsworth SJ, Van AT, et al. Comparison of readout-segmented echoplanar imaging (EPI) and single-shot EPI in clinical application of diffusion-weighted imaging of the pediatric brain. AJR Am J Roentgenol 2013;200:W437-43. 


\section{Legends}

FIG 1. Scatter diagram of apparent diffusion coefficient (ADC) values (expressed as $10^{-6}$ $\mathrm{mm}^{2} / \mathrm{s}$ ) for infantile hemangiomas $(H)$ and rhabdomyosarcomas $(R)$ along with mean and standard deviation.

FIG 2. A 6-month-old girl with a left orbital infantile hemangioma. A, Axial T2-weighted image showing a hyperintense mass with small internal flow voids. B, Axial T1-weighted image with contrast showing homogeneous enhancement. C, Axial ADC image showing hyperintense appearance corresponding to a measured ADC value of $1430 \times 10^{-6} \mathrm{~mm}^{2}$.

FIG 3. A 22-month-old girl with a right orbital rhabdomyosarcoma. A, Coronal T2-weighted image showing a hyperintense mass with small internal flow voids. B, Axial T1-weighted image with contrast showing homogeneous enhancement. C, Axial ADC image showing hypointense appearance corresponding to a measured ADC value of $585 \times 10^{-6} \mathrm{~mm}^{2}$. 
Table 1. Imaging appearance of orbital infantile hemangiomas and rhabdomyosarcomas

\begin{tabular}{lcccc}
\hline & T2W & T1W+C & T2W presence & ADC \\
& hyperintensity & homogeneous & of flow voids & hyperintensity \\
Infantile hemangioma, no. (\%) $[\mathrm{N}=21]$ & $21(100)$ & $21(100)$ & $21(100)$ & $21(100)$ \\
Rhabdomyosarcoma, no. (\%) $[\mathrm{N}=12]$ & $7(58)$ & $7(58)$ & $4(33)$ & $0(0)$ \\
\hline
\end{tabular}

$A D C$, apparent diffusion coefficient; $T 1 W+C$, contrast-enhanced T1-weighted; $T 2 W$, T2-weighted. 


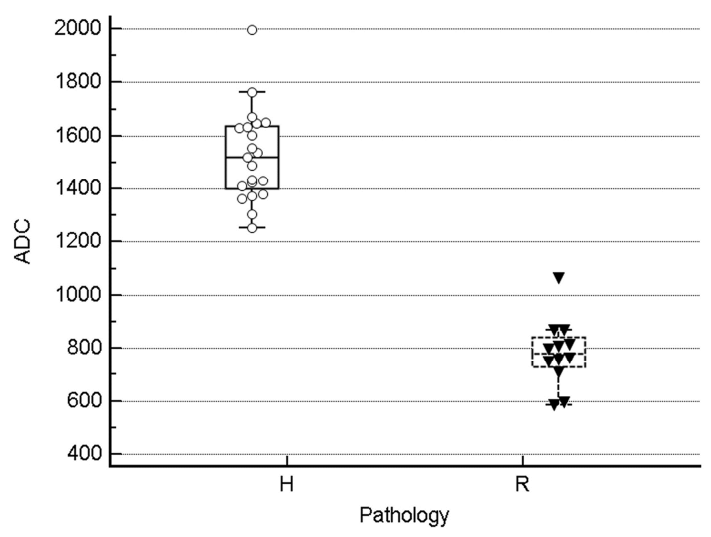




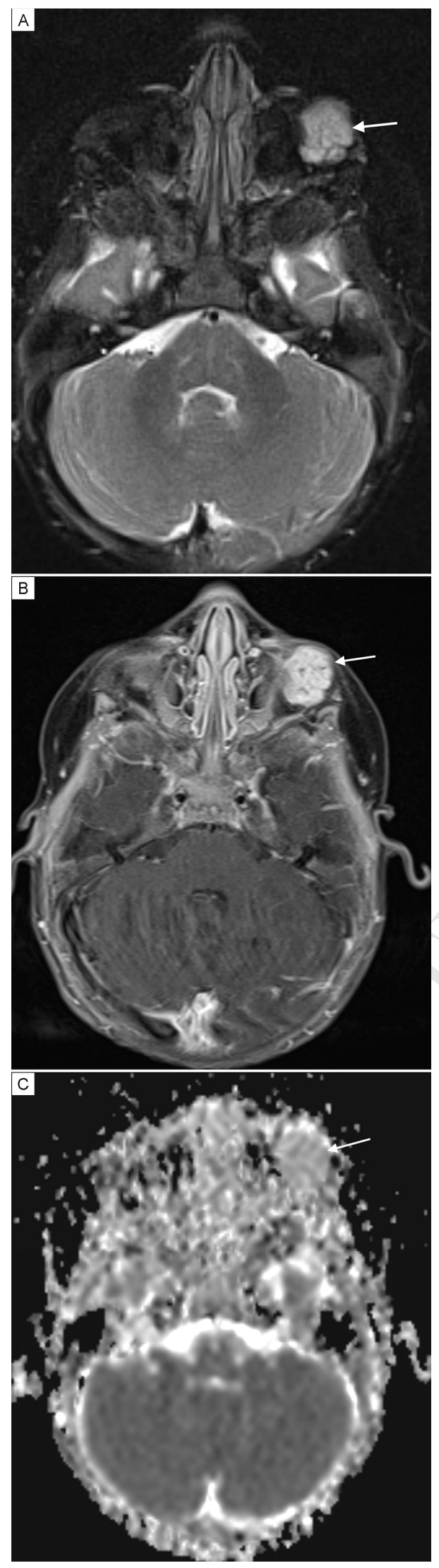



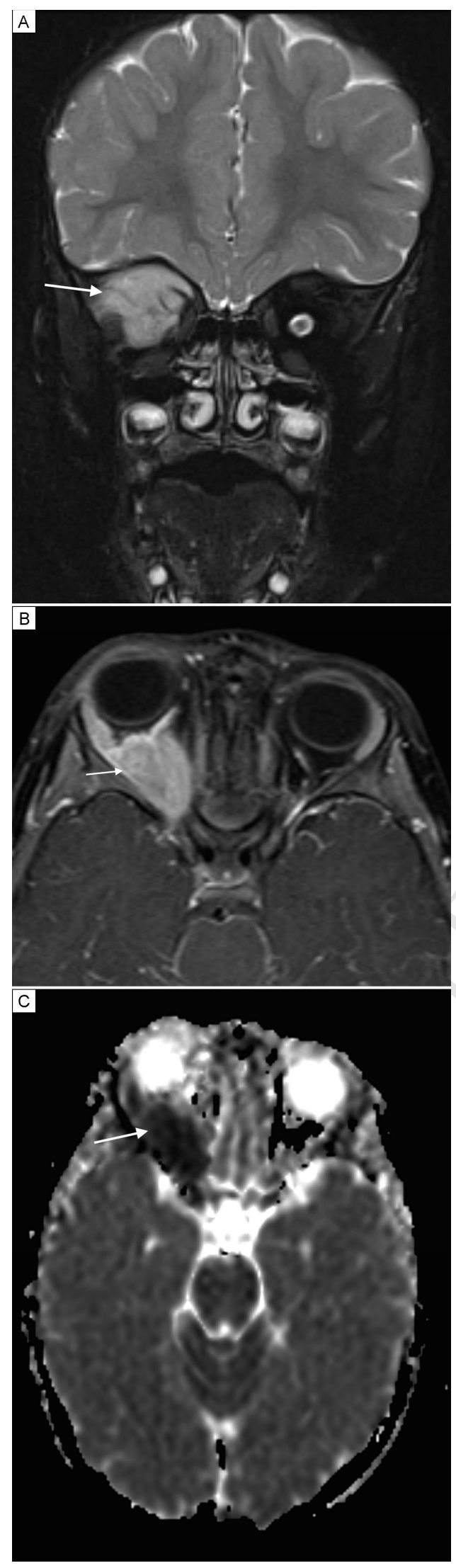